\title{
Butterfly fauna (Lepidoptera, Papilionoidea) in a heterogeneous area between two biodiversity hotspots in Minas Gerais, Brazil
}

\author{
Déborah Soldati ${ }^{1,3}$; Fernando Amaral da Silveira ${ }^{1,4}$ \& André Roberto Melo Silva ${ }^{2}$ \\ 1 Universidade Federal de Minas Gerais (UFMG), Instituto de Ciências Biológicas (ICB), Departamento de Zoologia, \\ Laboratório de Sistemática de Insetos. Belo Horizonte, MG, Brasil. \\ ${ }^{2}$ Centro Universitário UNA, Faculdade de Ciências Biológicas e da Saúde. Belo Horizonte, MG, Brasil. \\ ORCID: http://orcid.org/0000-0003-3113-5840. E-mail: andre.robertorml@gmail.com \\ ${ }^{3}$ ORCID: http://orcid.org/0000-0002-9546-2376. E-mail: desoldati@gmail.com (corresponding author). \\ ${ }^{4}$ ORCID: http://orcid.org/0000-0003-2408-2656. E-mail: f.a.silveira.ufmg@gmail.com
}

\begin{abstract}
This paper investigates the butterfly fauna of the 'Serra do Rola-Moça' State Park, Minas Gerais, Brazil. We evaluate i) the seasonal variation of species richness and composition; and ii) the variation in composition of the local butterfly assemblage among three sampling sites and between the dry and rainy seasons. Sampling was carried out monthly between November 2012 and October 2013, using entomological nets. After a total sampling effort of 504 net hours, 311 species were recorded. One of them is endangered in Brazil, and eight are probable new species. Furthermore, two species were new records for the region and eight considered endemic of the Cerrado domain. There was no significant difference in species richness between the dry and the rainy seasons, however the species composition varies significantly among sampling sites. Due to its special, heterogeneous environment, which is home to a rich butterfly fauna, its preservation is important for the conservation of the regional butterfly fauna.
\end{abstract}

Key-Words. Actinote quadra; Atlantic Rainforest; Butterfly richness; Cerrado; New records.

\section{INTRODUCTION}

The Atlantic Rainforest and the Cerrado domains present variable landscape structure (Tabarelli et al., 2005; Emery et al., 2006), being considered threatened and important biodiversity hotspots (Mittermeier et al., 2004). The heterogeneous vegetation in those floristic domains is habitat for a diverse fauna of butterflies (Emery et al., 2006). Although high butterfly species richness has been recorded in different areas of the Atlantic Rainforest and the Cerrado, as well as in transition areas between those domains, inventories in the various vegetation types in those ecosystems still need to be conducted (Carneiro et al., 2008).

The butterfly fauna of the Brazilian Cerrado is still poorly known, even in southern and southeastern Brazil, where many faunistic inventories have been done (Carneiro et al., 2008).

Some important butterfly inventories were done in the Cerrado in the Federal District and in the state of Minas Gerais (Brown Jr. \& Mielke, 1967a, 1967b, 1968; Emery et al., 2006; Mielke et al., 2008; Motta, 2002). Few inventories were focused on the butterfly fauna of non-urban fragments of Atlantic Rainforest in Minas Gerais (Brown Jr. \& Mielke, 1968; Brown Jr. \& Freitas, 2000; Ebert, 1969; Silva et al., 2010), although a significative portion of the Brazilian butterfly diversity occurs in this domain (Iserhard et al., 2018).

Many studies demonstrate that the number of active butterfly species decreases during the dry season, mainly due to scarcity of larval food (Brown Jr. \& Freitas, 2000). In the rainy season, when more species are active, adult females easily find new, soft, nutrient-reach leaves to feed the caterpillars (Iserhard et al., 2013). However, environmental characteristics, such as vegetation type, are more relevant than the temporal variation (dry and rainy seasons) in organizing species composition for Hesperiidae butterfly assemblages (Carneiro et al., 2014) and can also be relevant for the species composition of the other butterfly families.

The study of species diversity in different vegetation types is critical to planning and developing environmental protection and insect conservation actions (Lewinsohn et al., 2005), and faunistic inventories are indispensable tools for 
recording and describing the local biodiversity, as well as for management and protection of remnants of the Cerrado, of the Atlantic Rainforest and of the transition areas between them.

Here, the butterfly fauna of a heterogeneous area in the border of the Cerrado and the Atlantic Rainforest domains, in the Brazilian state of Minas Gerais, is inventoried and its species richness and composition in different vegetation types, in the dry and rainy seasons, are analyzed.

\section{MATERIAL AND METHODS}

\section{Study area}

The study was conducted in the 'Serra do Rola-Moça' State Park (PESRM) $\left(20^{\circ} 03^{\prime} 01^{\prime \prime} \mathrm{S}, 44^{\circ} 00^{\prime} 20^{\prime \prime} \mathrm{W}\right)$, located in the municipalities of Belo Horizonte, Brumadinho, Ibirité and Nova Lima in the state of Minas Gerais (Fig. 1). The park is in the region known as Quadrilátero Ferrífero ('Iron Quadrangle') in the southern Espinhaço Range, and is composed of different vegetation types of the Atlantic Rainforest domain (riparian and seasonal semi-deciduous forest) and of the Cerrado domain (cerrado sensu strictu [savanna], natural grasslands and rocky fields) (Drummond \& Martins, 2007).

The park is a mountainous area with altitudes raging between 1,000 m and 1,430 m (Drummond \& Martins, 2007), under a tropical climate, with a dry season be- tween April and September, and a rainy season between October and March, with an average total rainfall of $850 \mathrm{~mm}$ (Drummond \& Martins, 2007). Surrounding the park, there are mines and urban areas that directly affect the park's environment (Drummond \& Martins, 2007).

\section{Sampling and identification}

Sampling was conducted between November 2012 and October 2013, in twelve transects, two in each of six different vegetational types: rocky field, Cerrado sensu strictu (savanna) and natural grassland (physiognomies of the Cerrado); riparian forests and seasonal semi-deciduous forest (Atlantic Rainforest); and Forest-Cerrado transition areas. The twelve transects are $1 \mathrm{~km}$ to $1.5 \mathrm{~km}$ away from each other and were chosen considering their accessibility and vegetation types. Due to low temperatures and rain, the sampling effort was reduced in March.

Entomological nets were used to capture adult butterflies, following the standard procedures established by Iserhard (2011). Samples were carried out twice a month, with three collectors sampling for 3.5 hours along each of two transects each day, between 09:00 and 16:30.

The butterflies were sacrificed by thorax compression and stored in entomological envelopes for posterior identification. The following information was recorded for each captured butterfly: date, time, local of collection, and collector. The specimens collected were identified and deposited in the Centro de Coleções

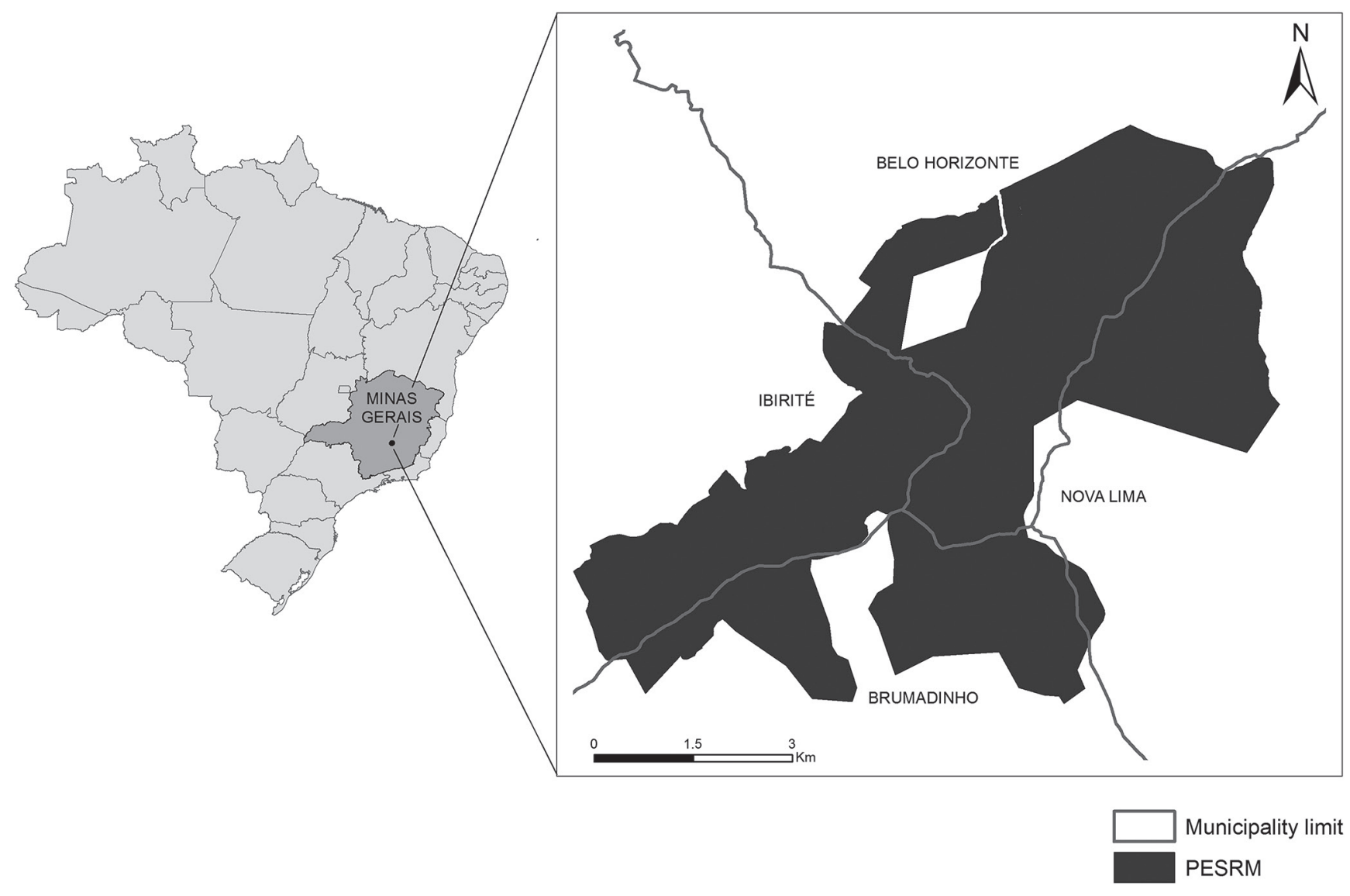

Figure 1. Location of the study site, the'Serra do Rola-Moça'State Park (PESRM), in Minas Gerais state, Brazil. 
Taxonômicas of the Universidade Federal de Minas Gerais (CCT-UFMG).

Specimen identification was accomplished with aid of the taxonomic literature (Brown Jr., 1992; Penz \& Devries, 2002; Willmott, 2003; Uehara-Prado et al., 2004; D'Abrera, 2005; Garwood et al., 2009; Santos et al., 2011), as well as of the illustrated list of South American butterflies (Warren et al., 2017). Specimens that could not be identified by the authors were taken for identification in the Laboratory of Studies of Neotropical Lepidoptera, of the Universidade Federal do Paraná (UFPR) (Hesperiidae), and Laboratory of Ecology and Systematics of Butterflies of the Universidade Estadual de Campinas (UNICAMP) (Riodinidae, Satyrinae and Ithomiinae [Nymphalidae]). The classification employed here were those of Lamas (2004); Wahlberg et al. (2009); for Hesperiidae, Warren et al. (2008, 2009); and for Riodinidae, Seraphim et al. (2018).

\section{Data analysis}

The Generalized Linear Model (GLM) was used to compare the species richness of the dry and rainy seasons, with richness being the response and seasons the explanatory variables. GLM was used to compare the species richness (response variable) of the sampling sites (explanatory variable). All GLM were submitted to residual analyses to evaluate adequacy of the error distribution (Crawley, 2013). Non-metric multidimensional scaling (NMDS) analyses employing the Jaccard similarity index were employed to compare the species composition between seasons and between sampling sites. Analyses of similarities (ANOSIM) were performed to test for differences between seasons and sampling sites. All analyses were performed in R software (R Core Team, 2014) using gdata, vegan and RT4Bio packages. Sample coverage of species richness in each of the dry and rainy seasons and in both seasons were verified using the RStudio software (RStudio Team, 2015) with vegan, iNEXT, ggplot2, devtools, gridExtra and grid packages.

\section{RESULTS AND DISCUSSION}

\section{Species richness and composition}

After a total sampling effort of 504 net hours, 1,638 individuals of 311 species were recorded. The richest family was Hesperiidae with 113 species (36.5\%), followed by Nymphalidae with 98 species (31.5\%), Riodinidae with 31 (10\%), Lycaenidae with 29 (9.5\%), Pieridae with 24 (7.5\%) and Papilionidae with 16 species (5\%) (Table 1).

The number of species of Pieridae and Papilionidae may have been underestimated, because observed individuals were hard to catch with entomological nets. Moreover, the sampling period (between 09:00 and16:30) may have also influenced the number of species recorded for some taxa, as Brassolini (Nymphalidae) and Hesperiidae, which include some typically crepuscu- lar species (Mielke \& Casagrande, 1997) that were rarely sampled.

In the rainy season (October to March), 230 species were recorded, of which, 102 were sampled only in that season. In the dry season (April to September), 209 species were recorded, 81 of them unique to that season. A total of 128 species were sampled in both seasons. Sample coverage of species richness, in each season, reached almost $70 \%$ and indicates that more species could have been sampled (Figs. 2, 3). The sampling cov-

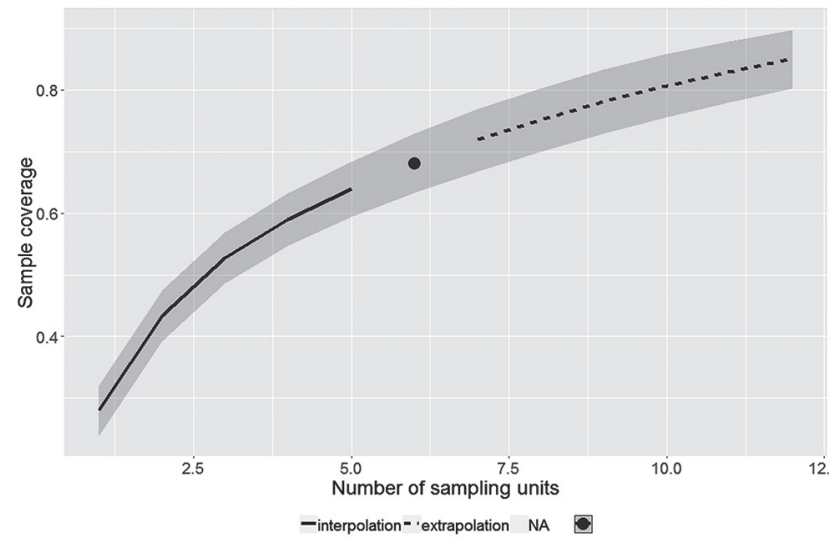

Figure 2. Sample coverage of species richness in rainy season, in the'Serra do Rola-Moça' State Park, Minas Gerais state, Brazil.

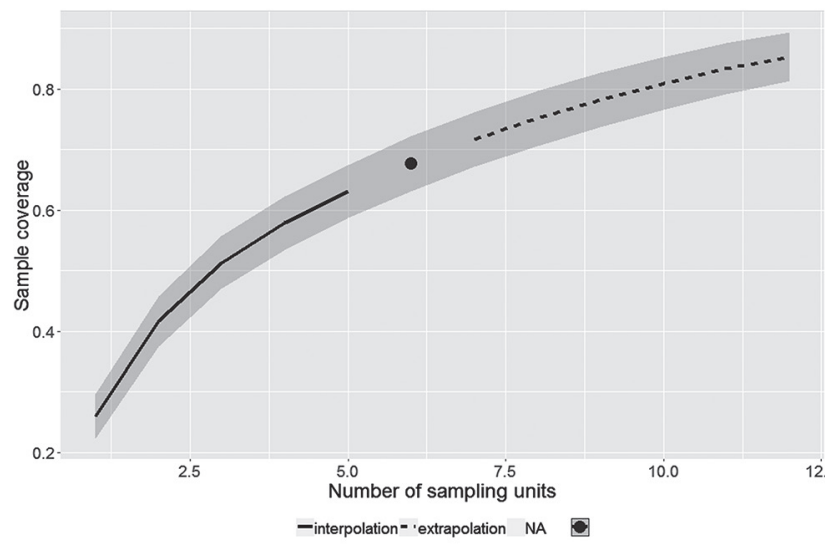

Figure 3. Sample coverage of species richness in dry season, in the 'Serra do Rola-Moça' State Park, Minas Gerais state, Brazil.

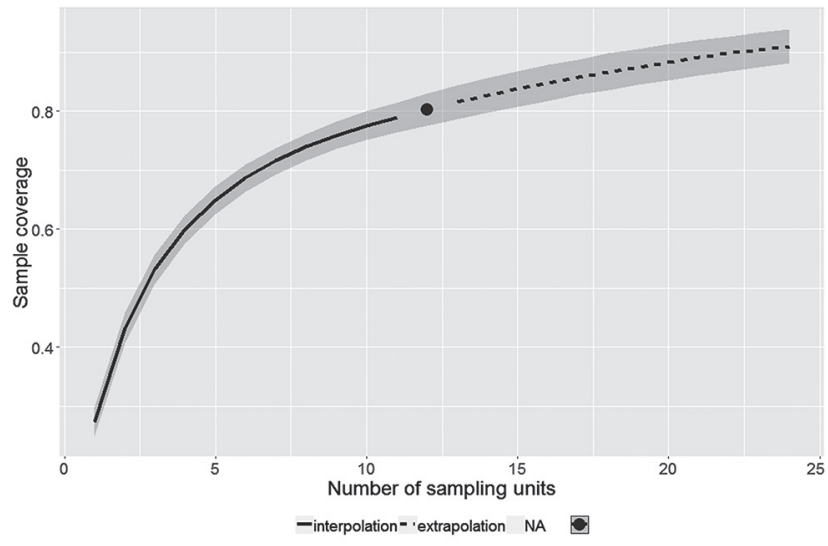

Figure 4. Sample coverage of species richness in rainy and dry seasons, in the 'Serra do Rola-Moça' State Park, Minas Gerais state, Brazil. 
Table 1. Species collected in the dry and rainy seasons in environments of Cerrado domain (c), Atlantic Rainforest (f) and transition between Cerrado and Atlantic Rainforest (t). ${ }^{*}=$ new record for the Quadrilátero Ferrifero; ${ }^{*}=$ new record for the state of Minas Gerais; $\bullet=$ endemic species of the Cerrado. Numbers in parenthesis after suprageneric taxa are the numbers of species sampled in each taxon.

\begin{tabular}{|c|c|c|c|c|c|c|c|}
\hline Taxa & $\begin{array}{c}\text { Sampling } \\
\text { environment }\end{array}$ & $\begin{array}{c}\text { Dry } \\
\text { season }\end{array}$ & $\begin{array}{c}\text { Rainy } \\
\text { season }\end{array}$ & Taxa & $\begin{array}{c}\text { Sampling } \\
\text { environment }\end{array}$ & $\begin{array}{c}\text { Dry } \\
\text { season }\end{array}$ & $\begin{array}{l}\text { Rainy } \\
\text { season }\end{array}$ \\
\hline HESPERIIDAE (113) & & & & Aguna albistria albistria (Plötz, 1880) & c & & $x$ \\
\hline Hesperiinae $(50)$ & & & & Aguna asander asander (Hewitson, 1867) & $c, f$ & & $x$ \\
\hline Anthoptus epictetus (Fabricius, 1793) & $\mathbf{t}$ & $x$ & $x$ & Anastrus sempiternus simplicior (Möschler, 1877) & $\mathbf{t}$ & & $x$ \\
\hline Artines aquilina (Plötz, 1882) & $\mathbf{t}$ & $x$ & & Anisochoria superior Mabille, 1898 & f & & $x$ \\
\hline Callimormus interpunctata (Plötz, 1884) & $\mathbf{t}$ & & $x$ & Antigonus erosus (Hübner, [1812]) & $c, f, t$ & & $X$ \\
\hline Callimormus saturnus (Herrich-Schäffer, 1869) & $f, t$ & $x$ & $x$ & Antigonus liborius areta Evans, 1953 & $f, t$ & $x$ & $x$ \\
\hline Chalcone briquenydan chalcone (Schaus, 1902) & $\mathbf{t}$ & & $x$ & Astraptes fulgerator fulgerator (Walch, 1775) & f & $x$ & \\
\hline Chalcone sp.n & $\mathbf{t}$ & & $x$ & Astraptes naxos (Hewitson, 1867) & $\mathbf{t}$ & & $x$ \\
\hline Chalcone zisa (Plötz, 1882) & $\mathbf{t}$ & $x$ & & Celaenorrhinus similis Hayward, 1933 & $\mathbf{t}$ & & $x$ \\
\hline Cobalopsis miaba (Schaus, 1902) & $f, t$ & $X$ & & Chioides catillus catillus (Cramer, 1779) & $\mathbf{t}$ & $X$ & $X$ \\
\hline Cobalopsis sp. & $\mathbf{t}$ & $x$ & & Chiomara asychis autander (Mabille, 1891) & $c, f, t$ & $x$ & $x$ \\
\hline Corticea mendica mendica (Mabille, 1898) & $\mathbf{t}$ & & $x$ & Chiomara basigutta (Plötz, 1884) & c & & $x$ \\
\hline Cumbre belli eberti Evans, 1955 & $\mathbf{t}$ & $x$ & & Cogia abdul Hayward, 1947 & c & $x$ & \\
\hline Cumbresp.n & $\mathbf{t}$ & & $x$ & Cogia calchas (Herrich-Schäffer, 1869) & $c, f, t$ & $X$ & $X$ \\
\hline Cymaenes gisca Evans, 1955 & $f, t$ & $X$ & $X$ & Cogia cerradicola (Mielke, 1967)• & c & & $X$ \\
\hline Cymaenes sp. & $c, t$ & $X$ & & Cogia grandis Riley, $1921 \cdot$ & c & & $X$ \\
\hline Cymaenes sp.n & $\mathbf{t}$ & $x$ & & Cogia hassan hassan Butler, 1870 & c & $x$ & \\
\hline Decinea lucifer (Hübner, [1831]) & $\mathbf{t}$ & & $x$ & Cogia punctilia Plötz, 1882 & c & $x$ & \\
\hline Gallio carasta (Schaus, 1902) & $\mathbf{t}$ & & $x$ & Cogia sp. Butler, 1870 & c & & $X$ \\
\hline Hylephila phyleus phyleus (Drury, 1773) & $c, t$ & & $x$ & Cogia sp.n & c & $x$ & $x$ \\
\hline Justinia kora (Hewitson, 1877) & $\mathbf{t}$ & $x$ & & Diaeus lacaena (Hewitson, 1869) & f & $x$ & \\
\hline Lamponia elegantula (Herrich-Schäffer, 1869) & f & $x$ & & Elbella luteizona (Mabille, 1877) & c & & $x$ \\
\hline Lerema duroca duroca (Plötz, 1882) & f & & $x$ & Gesta gesta (Herrich-Schäffer, 1863) & $f, t$ & $x$ & \\
\hline Lerema veadeira Mielke, 1968 • & c & $X$ & $X$ & Gorgythion begga begga (Prittwitz, 1868) & $c, f, t$ & $X$ & $X$ \\
\hline Lerodea erythrostictus (Prittwitz, 1868) & $c, t$ & $X$ & & Heliopetes alana (Reakirt, 1868) & $f, t$ & $x$ & \\
\hline Lerodea eufala eufala (W.H. Edwards, 1869) & c & $x$ & $x$ & Heliopetes arsalte (Linnaeus, 1758) & $c, t$ & $x$ & $x$ \\
\hline Levina levina (Plötz, 1884) & $c, t$ & $X$ & $x$ & Heliopetes macaira obrigera (Mabille, 1888) & $f, t$ & $X$ & $X$ \\
\hline Libra anatolica (Plötz, 1883) & $\mathbf{t}$ & & $x$ & Heliopetes ochroleuca J. Zikán, 1938 & f & $x$ & \\
\hline Lucida lucia lucia (Capronnier, 1874) & $f, t$ & $X$ & $X$ & Heliopetes omrina (Butler, 1870) & $c, t$ & $X$ & $X$ \\
\hline Lucida sp. & $\mathbf{t}$ & $x$ & & Heliopetes petrus (Hübner, [1819]) & $\mathbf{t}$ & $x$ & $x$ \\
\hline Lucida sp.n & $\mathbf{t}$ & $x$ & & Heliopyrgus domicella willi (Plötz, 1884) & $c, f$ & $x$ & \\
\hline Miltomiges cinnamomea (Herrich-Schäffer, 1869) & $f, t$ & $X$ & & Milanion leucaspis (Mabille, 1878) & $f, t$ & $X$ & $X$ \\
\hline Nastra dryas (Hayward, 1940) & f & $X$ & & Morvina fissimacula fissimacula (Mabille, 1878) & f & & $X$ \\
\hline Niconiades merenda (Mabille, 1878) & $\mathbf{t}$ & $x$ & & Mylon maimon (Fabricius, 1775) & f & $X$ & \\
\hline Panoquina hecebolus (Scudder, 1872) & $\mathbf{t}$ & & $x$ & Nisoniades macarius (Herrich-Schäffer, 1870) & f & & $x$ \\
\hline Panoquina peraea (Hewitson, 1866) * & $\mathbf{t}$ & $x$ & & Oechydrus chersis evelinda (Butler, 1870) & f & $x$ & \\
\hline Pheraeus argynnis (Plötz, 1884) & $\mathbf{t}$ & & $x$ & Phocides polybius phanias (Burmeister, 1880) & c & & $x$ \\
\hline Phlebodes sp.n & $\mathbf{t}$ & & $X$ & Polyctor polyctor polyctor (Prittwitz, 1868) & $f, t$ & & $X$ \\
\hline Polites vibex catilina (Plötz, 1886) & $c, t$ & $x$ & & Pyrgus orcus (Stoll, 1780) & $c, f, t$ & $x$ & $x$ \\
\hline Pompeius dares (Plötz, 1883) & f & $x$ & & Pyrgus orcynoides (Giacomelli, 1928) & c & & $x$ \\
\hline Pompeius pompeius (Latreille, [1824]) & $\mathbf{t}$ & & $x$ & Pyrrhopyge charybdis charybdis Westwood, 1852 & $c, t$ & & $X$ \\
\hline Pompeius postpuncta (Draudt, 1923) & c & $x$ & $x$ & Pyrrhopyge charybdis semita Evans, 1951 & c & & $x$ \\
\hline Remella remus (Fabricius, 1798) & $\mathbf{t}$ & $x$ & $x$ & Pyrrhopygesp. & c & & $x$ \\
\hline Thespieus homochromus Mielke, 1978 & c & $x$ & $x$ & Pythonides lancea (Hewitson, 1868) & f & $x$ & $x$ \\
\hline Vehilius clavicula (Plötz, 1884) & $\mathbf{t}$ & $X$ & & Quadrus cerialis (Stoll, 1782) & $\mathbf{t}$ & & $X$ \\
\hline Vettius artona (Hewitson, 1868) & f & $X$ & & Quadrus u-Iucida mimus (Mabille and Boullet, 1912) & f & $X$ & \\
\hline Vettius diversa diversa (Herrich-Schäffer, 1869) & $\mathbf{t}$ & $x$ & $x$ & Sarbia catomelaena Mabille and Boullet, 1908 • & c & & $x$ \\
\hline Vettius lucretius (Latreille, [1824]) & f & $X$ & $X$ & Sophista latifasciata (Spitz, 1930) • & $c, t$ & $X$ & $X$ \\
\hline Vettius marcus (Fabricius, 1787) & $f, t$ & $x$ & $x$ & Sostrata bifasciata bifasciata (Ménétriés, 1829) & $\mathbf{t}$ & $x$ & \\
\hline Vidius nostra nostra Evans, 1955 & c & $X$ & & Sostrata cronion (C. Felder \& R. Felder, 1867) & f & $X$ & \\
\hline Vidius sp. Evans, 1955 & $\mathbf{t}$ & $X$ & & Theagenes dichrous (Mabille, 1878) & f & & $X$ \\
\hline Vinius letis (Plötz, 1883) & $\mathbf{t}$ & $x$ & $x$ & Trina geometrina (C. Felder \& R. Felder, 1867) & $f, t$ & $x$ & $x$ \\
\hline Pyrginae (63) & & & & Urbanus chalco (Hübner, 1823) & f & $x$ & \\
\hline Achlyodes busirus rioja Evans, 1953 & f & $X$ & & Urbanus dorantes (Stoll, 1790) & $f, t$ & $X$ & $x$ \\
\hline
\end{tabular}




\begin{tabular}{|c|c|c|c|}
\hline Taxa & $\begin{array}{c}\text { Sampling } \\
\text { environment }\end{array}$ & $\begin{array}{c}\text { Dry } \\
\text { season }\end{array}$ & $\begin{array}{c}\text { Rainy } \\
\text { season }\end{array}$ \\
\hline Urbanus esta Evans, 1952 & $\mathbf{t}$ & & $x$ \\
\hline Urbanus pronta Evans, 1952 & $f, t$ & & $x$ \\
\hline Urbanus teleus (Hübner, 1821) & $\mathbf{t}$ & & $x$ \\
\hline Urbanus virescens (Mabille, 1877) & $\mathbf{t}$ & & $x$ \\
\hline Viola sp. & f & $x$ & \\
\hline Viola viollela (Mabille, 1898) & $\mathbf{t}$ & & $x$ \\
\hline Zopyrion evenor evenor Godman, 1901 & $c, t$ & $x$ & $x$ \\
\hline Zopyrion evenor thania Godman, 1901 & $\mathbf{t}$ & & $x$ \\
\hline \multicolumn{4}{|l|}{ NYMPHALIDAE (98) } \\
\hline \multicolumn{4}{|l|}{ Apaturinae (1) } \\
\hline Doxocopa laurentia (Godart, [1824]) & $c, f$ & $x$ & $x$ \\
\hline \multicolumn{4}{|l|}{ Biblidinae (17) } \\
\hline Callicore sorana sorana (Godart, [1824]) & $c, f, t$ & $x$ & $x$ \\
\hline Cybdelis phaesyla (Hübner, [1831]) & $f, t$ & $x$ & $x$ \\
\hline Diaethria clymena (Cramer, 1775) & $f, t$ & $x$ & $x$ \\
\hline Dynamine aerata (Butler, 1877) & $f, t$ & $x$ & $x$ \\
\hline Dynamine agadles agacles (Dalman, 1823) & $\mathbf{t}$ & $x$ & $x$ \\
\hline Dynamine postverta postverta (Cramer, 1779) & $f, t$ & $x$ & $x$ \\
\hline Dynamine tithia (Hübner, 1823) & $\mathbf{t}$ & & $x$ \\
\hline Ectima thecla (Fabricius, 1796) & $f, t$ & $x$ & \\
\hline Epiphile hubneri Hewitson, 1861 & $f, t$ & & $x$ \\
\hline Epiphile orea orea (Hübner, [1823]) & f & & $x$ \\
\hline Eunica volumna volumna (Godart, [1824]) & $f, t$ & $x$ & $x$ \\
\hline Hamadryas epinome (C. Felder \& R. Felder, 1867) & $f, t$ & $x$ & $x$ \\
\hline Hamadryas laodamia (Cramer, 1777) & f & $x$ & \\
\hline Marpesia chiron (Fabricius, 1775) & $f, t$ & $x$ & $x$ \\
\hline Marpesia petreus (Cramer, 1776) & f & $x$ & \\
\hline Myscelia orsis (Drury, 1782) & $f, t$ & $x$ & $x$ \\
\hline Nica flavilla (Godart, [1824]) & f & $x$ & \\
\hline \multicolumn{4}{|l|}{ Charaxinae (4) } \\
\hline Archaeoprepona chalciope (Hübner, [1823]) & $\mathbf{t}$ & & $x$ \\
\hline Fountainea ryphea (Cramer, 1775) & $\mathbf{t}$ & $x$ & \\
\hline Memphis moruus stheno (Prittwitz, 1865) & f & & $x$ \\
\hline Memphis otrere (Hubner, [1825]) & $c, f$ & $x$ & $x$ \\
\hline \multicolumn{4}{|l|}{ Danainae (20) } \\
\hline Aeria elara (Hewitson, 1855) & $f, t$ & $x$ & $x$ \\
\hline Aeria olena olena Weymer, 1875 & $f, t$ & $x$ & $x$ \\
\hline Danaus gilippus (Cramer, 1775) & f & $x$ & \\
\hline Danaus plexippus (Linnaeus, 1758) & $c, t$ & $x$ & $x$ \\
\hline Dircenna dero (Hübner, 1823) & $f, t$ & $x$ & $x$ \\
\hline Episcada carcinia Schaus, 1902 & $\mathbf{t}$ & & $x$ \\
\hline Episcada hymenaea (Prittwitz, 1865) & $f, t$ & $x$ & $x$ \\
\hline Hypoleria lavinia (Hewitson, [1855]) & f & $x$ & \\
\hline Hypothyris euclea laphria (Doubleday, 1847) & f & $x$ & \\
\hline Hypothyris ninonia daeta (Boisduval, 1836) & $f, t$ & $x$ & $x$ \\
\hline Ithomia agnosia Hewitson, [1855] & $f, t$ & $x$ & $x$ \\
\hline Lycorea halia (Hübner, 1816) & $f, t$ & & $x$ \\
\hline Mcclungia cymo salonina (Hewitson, 1855) & $\mathbf{t}$ & & $x$ \\
\hline Mechanitis lysimnia lysimnia (Fabricius, 1793) & $f, t$ & $X$ & $X$ \\
\hline Mechanitis polymnia casabranca Haensch, 1905 & $f, t$ & $x$ & $x$ \\
\hline Oleria aquata (Weymer, 1875) & $f, t$ & $x$ & $x$ \\
\hline Pseudoscada acilla quadrifasciata Talbot, 1928 • & $\mathbf{t}$ & $X$ & \\
\hline Pseudoscada erruca (Hewitson, 1855) & $c, f, t$ & $x$ & $x$ \\
\hline Pteronymia carlia Schaus, 1902 & f & $x$ & \\
\hline Thyridia psidii (Linnaeus, 1758) & $f, t$ & $x$ & $x$ \\
\hline \multicolumn{4}{|l|}{ Heliconiinae (13) } \\
\hline Actinote parapheles Jordan, 1913 & $f, t$ & $x$ & $x$ \\
\hline
\end{tabular}

\begin{tabular}{|c|c|c|c|}
\hline Taxa & $\begin{array}{c}\text { Sampling } \\
\text { environment }\end{array}$ & $\begin{array}{c}\text { Dry } \\
\text { season }\end{array}$ & $\begin{array}{l}\text { Rainy } \\
\text { season }\end{array}$ \\
\hline Actinote quadra (Schaus, 1902) & c & & $x$ \\
\hline Actinote surima surima (Schaus, 1902) & c & $x$ & $x$ \\
\hline Actinote thalia pyrrha (Fabricius, 1775) & $c, f, t$ & $x$ & $x$ \\
\hline Agraulis vanillae (Linnaeus, 1758) & $c, f, t$ & $x$ & $x$ \\
\hline Dione juno juno (Cramer, 1779) & c & & $x$ \\
\hline Dryadula phaetusa (Linnaeus, 1758) & $c, t$ & $x$ & $X$ \\
\hline Dryas iulia (Fabricius, 1775) & $f, t$ & $x$ & $x$ \\
\hline Eueides pavana Ménétriés, 1857 & $f, t$ & $x$ & $x$ \\
\hline Heliconius besckei Ménétriés, 1857 & $c, f, t$ & $x$ & $x$ \\
\hline Heliconius erato phyllis (Fabricius, 1775) & $c, f, t$ & $x$ & $x$ \\
\hline Heliconius ethilla (Godart, 1819) & $f, t$ & $x$ & $x$ \\
\hline Philaethria wernickei (Röber, 1906) & f & $x$ & \\
\hline \multicolumn{4}{|l|}{ Limenitidinae (6) } \\
\hline Adelpha calliphane Fruhstorfer, 1915 & f & & $x$ \\
\hline Adelpha gavina Fruhstorfer, 1915 & $\mathbf{t}$ & & $x$ \\
\hline Adelpha plesaure Hübner, 1823 & $f, t$ & $x$ & $x$ \\
\hline Adelpha serpa serpa (Boisduval, 1836) & f & & $x$ \\
\hline Adelpha syma (Godart, [1824]) & $c, f, t$ & $x$ & $x$ \\
\hline Adelpha thessalia (C. Felder \& R. Felder, 1867) & $\mathbf{t}$ & $x$ & \\
\hline \multicolumn{4}{|l|}{ Nymphalinae (9) } \\
\hline Colobura dirce (Linnaeus, 1758) & $f, t$ & $x$ & $x$ \\
\hline Eresia lansdorfi (Godart, 1819) & $c, f, t$ & $x$ & $x$ \\
\hline Junonia evarete (Cramer, 1779) & $c, t$ & $x$ & $x$ \\
\hline Siproeta epaphus (Latreille, [1813]) & $f, t$ & $x$ & $x$ \\
\hline Siproeta stelenes meridionalis (Fruhstorfer, 1909) & $f, t$ & $X$ & $x$ \\
\hline Tegosa claudina (Eschscholtz, 1821) & $c, f, t$ & $x$ & $x$ \\
\hline Telenassa teletusa (Godart, [1824]) & $f, t$ & $x$ & $x$ \\
\hline Vanessa braziliensis (Moore, 1883) & c & $X$ & $x$ \\
\hline Vanessa myrinna (Doubleday, 1849) & $c, f, t$ & $x$ & $x$ \\
\hline \multicolumn{4}{|l|}{ Satyrinae (28) } \\
\hline Antirrhea archaea (Hübner, [1822]) & f & & $x$ \\
\hline Biblis hyperia (Cramer, 1779) & $f, t$ & $x$ & $x$ \\
\hline Blepolenis batea (Hübner, [1821]) & $\mathbf{t}$ & & $x$ \\
\hline Caligo illioneus (Cramer, 1775) & $\mathbf{t}$ & $x$ & \\
\hline Capronnieria galesus (Godart, [1824]) & f & $x$ & \\
\hline Eryphanis reevesii reevesii (Doubleday, [1849]) & $\mathbf{t}$ & $x$ & \\
\hline Godartiana muscosa (Butler, 1870) & $f, t$ & $x$ & $x$ \\
\hline Hermeuptychia atalanta (Butler, 1867) & f & $x$ & \\
\hline Hermeuptychia gisella (Hayward, 1957) & f & $x$ & \\
\hline Hermeuptychia sp. & f & $X$ & \\
\hline Moneuptychia itapeva Freitas, 2007 & $c, t$ & $x$ & $x$ \\
\hline Moneuptychia paeon (Godart, [1824]) & $\mathbf{t}$ & & $x$ \\
\hline Moneuptychia soter (Butler, 1877) & $\mathbf{t}$ & $x$ & $x$ \\
\hline Moneuptychia sp.n & f & $x$ & \\
\hline Morpho anaxibia (Esper, [1801]) & c & & $x$ \\
\hline Morpho helenor (Cramer, 1776) & $f, t$ & $x$ & $x$ \\
\hline Morpho menelaus (Linnaeus, 1758) & $f, t$ & $x$ & $x$ \\
\hline Paryphthimoides grimon (Godart, [1824]) & $\mathbf{t}$ & & $x$ \\
\hline Paryphthimoides eous (Butler, 1867) & $\mathbf{t}$ & $x$ & \\
\hline Paryphthimoides poltys (Prittwitz, 1865) & $f, t$ & $x$ & \\
\hline Pharneuptychia innocentia (C. Felder \& R. Felder, 1867) & $c, t$ & $x$ & $x$ \\
\hline Pharneuptychia sp. & $c, t$ & $x$ & $x$ \\
\hline Yphthimoides affinis (Butler, 1867) & f & $X$ & \\
\hline Yphthimoides angularis (Butler, 1867) & $\mathbf{t}$ & $x$ & $x$ \\
\hline Yphthimoides manasses (C. Felder \& R. Felder, 1867) & c & & $x$ \\
\hline Yphthimoides pacta (Weymer, 1911) & c & & $x$ \\
\hline Yphthimoides saltuensis (Hayward, 1962) & $c, f, t$ & $x$ & $x$ \\
\hline
\end{tabular}




\begin{tabular}{cccc}
\hline Taxa & $\begin{array}{c}\text { Sampling } \\
\text { environment }\end{array}$ & $\begin{array}{c}\text { Dry } \\
\text { season }\end{array}$ & $\begin{array}{c}\text { Rainy } \\
\text { season }\end{array}$ \\
\hline Yphthimoides straminea $($ Butler, 1867) • & $\mathrm{f}$ & $\mathrm{X}$
\end{tabular}

Yphthimoides straminea (Butler, 1867) •

\section{RIODINIDAE (31)}

\section{Riodininae (31)}

Baeotis johannae cearaica Seitz, 1916

Baeotis melanis Hübner, [1831])

Barbicornis basilis melanops Butler, 1873

Calephelis sp.

Caria plutargus (Fabricius, 1793)

Chalodeta theodora (C. Felder \& R. Felder, 1862)

Charis cadytis Hewitson, 1866

Chorinea licursis (Fabricius, 1775)

Dachetola azora (Godart, [1824])

Emesis diogenia Prittwitz, 1865

Emesis ocypore (Geyer, 1837)

Emesis russula Stichel, 1910

\section{Emesis sp.}

Eurybia elvina elvina Stichel, 1910

Eurybia misellivestis Stichel, 1910

Eurybia pergaea (Geyer, 1832)

Lasaia agesilas (Latreille, [1809])

Lemonias stalachtioides (Butler, 1867)

Leucochimona icare matatha (Hewitson, 1873)

Melanis xenia xenia (Hewitson, [1853])

Mesene sp.

Mesosemia odice (Godart, [1824])

Metacharis ptolomaeus (Fabricius, 1793)

Notheme erota agathon (C. Felder \& R. Felder, 1865)

Panara soana bacana Callaghan, 1997

Pirascca sagaris (Cramer, 1775)

Riodina lycisca (Hewitson, [1853])

Stalachtis phlegia susanna (Fabricius, 1787)

Stichelia bocchoris (Hewitson, 1876)

Synargis calyce (C. Felder \& R. Felder, 1862)

Synargis paulistina (Stichel, 1910)

\section{LYCAENIDAE (29)}

Polyommatinae (3)

Hemiargus hanno (Stoll, 1790)

Leptotes cassius (Cramer, 1775)

Zizula cyna (W.H. Edwards, 1881)

Theclinae (26)

Allosmaitia strophius (Godart, [1824])

Arawacus ellida (Hewitson, 1867)

Arawacus meliboeus (Fabricius, 1793)

Arawacus tarania (Hewitson, 1868)

Aubergina vanessoides (Prittwitz, 1865)

Brangas getus (Fabricius, 1787)

Brangas torfrida (Hewitson, 1867)

Calycopis sp. 1

Calycopis sp.2

Cyanophrys sp.

Erora opisena (H.H. Druce, 1912)

Ministrymon cruenta (Gosse, 1880)

Nicolaea cauter (H.H. Druce, 1907)

Nicolaea schausa (Jones, 1912)

Nicolaea sp.

Ocaria ocrisia (Hewitson, 1868)

\begin{tabular}{|c|c|}
\hline $\mathbf{t}$ & $X$ \\
\hline $\mathbf{t}$ & $X$ \\
\hline f & \\
\hline$c, f, t$ & $x$ \\
\hline $\mathbf{t}$ & \\
\hline $\mathbf{t}$ & \\
\hline$f, t$ & $x$ \\
\hline c & \\
\hline $\mathbf{t}$ & $x$ \\
\hline $\mathbf{t}$ & $x$ \\
\hline t & \\
\hline$t$ & \\
\hline$t$ & $X$ \\
\hline f & $x$ \\
\hline f & $x$ \\
\hline$f$ & $x$ \\
\hline $\mathbf{t}$ & \\
\hline c & $X$ \\
\hline $\mathbf{t}$ & \\
\hline$f$ & $x$ \\
\hline$f$ & $x$ \\
\hline $\mathbf{t}$ & $x$ \\
\hline $\mathbf{t}$ & \\
\hline $\mathbf{t}$ & \\
\hline $\mathbf{t}$ & \\
\hline $\mathbf{t}$ & $X$ \\
\hline $\mathbf{t}$ & \\
\hline $\mathbf{t}$ & \\
\hline $\mathbf{t}$ & $X$ \\
\hline $\mathbf{t}$ & $X$ \\
\hline
\end{tabular}

(Stoll, 178

Rekoa marius (Lucas, 1857)

Rekoa palegon (Cramer, 1780)

Strymon azuba (Hewitson, 1874)

Strymon bazochii (Godart, [1824])

Strymon bubastus (Stoll, 1780)

Strymon lucena (Hewitson, 1868)

Strymon mulucha (Hewitson, 1867)

Strymon sp.

Thereus cithonius (Godart, [1824])

\section{PIERIDAE (24)}

Coliadinae (15)

Anteos menippe (Hübner, [1818])

Aphrissa statira (Cramer, 1777)

Eurema albula (Cramer, 1775) (undentified subespecies)

Eurema albula sinoe (Godart, 1819)

Eurema arbela Geyer, 1832

Eurema elathea flavescens (Chavannes, 1850)

Eurema phiale paula (Röber, 1909)

Leucidia brephos (Hübner, [1809])

Leucidia elvina (Godart, 1819)

Phoebis argante (Fabricius, 1775)

Phoebis neocypris (Hübner, [1823])

Phoebis sennae sennae (Linnaeus, 1758)

Pyrisitia leuce (Boisduval, 1836)

Pyrisitia nise tenella (Boisduval, 1836)

Rhabdodryas trite banksi (Breyer, 1939)

Dismorphiinae (3)

Dismorphia amphione astynome (Dalman, 1823)

Dismorphia thermesia thermesia (Godart, 1819)

Pseudopieris nehemia nehemia (Boisduval, 1836)

Pierinae (6)

Archonias bassolis tereas (Godart, 1819)

Glutophrissa drusilla drusilla (Cramer, 1777)

Hesperocharis anguitia anguitia (Godart, 1819)

Hesperocharis leucania (Boisduval, 1836)

Melete lycimnia flippantha (Fabricius, 1793)

Pereute antodyca (Boisduval, 1836)

\section{PAPILIONIDAE (16)}

Papilioninae (16)

Battus polydamas (Linnaeus, 1758)

Eurytides dolicaon (Cramer, 1775)

Heraclides anchisiades capys (Hübner, [1809])

Heraclides astyalus (Godart, 1819)

Heraclides hectorides (Esper, 1794)

Heraclides thoas brasiliensis (Rothschild \& Jordan, 1906)

Heraclides torquatus polybius (Swainson, 1823)

Mimoides lysithous lysithous (Hübner, 1821)

Parides agavus (Drury, 1782)

Parides anchises nephalion (Godart, 1819)

Parides bunichus diodorus (Hopffer, 1865) •

Parides neophilus eurybates (G. Gray, [1853])

Parides proneus (Hübner, [1831])

Parides sesostris (Cramer, 1779)

Protesilaus helios (Rothschild \& Jordan, 1906)

Pterourus scamander scamander (Boisduval, 1836)

$\begin{array}{ccc}\text { Sampling } & \text { Dry } & \text { Rainy } \\ \text { environment } & \text { season } & \text { season }\end{array}$

$\begin{array}{ccc}\text { c } & & X \\ \mathbf{c} & & X \\ \mathbf{t} & X & \\ \mathbf{c} & X & \\ \mathbf{c} & & X \\ \mathbf{c} & X & X \\ \mathbf{c} & X & \\ \mathbf{t} & & X \\ \text { c, t } & & X \\ \mathbf{t} & & X\end{array}$

$\begin{array}{ccc}\text { t } & & X \\ \text { c, t } & & X \\ \text { c, f, t } & X & X \\ \text { c, f, t } & X & X \\ \mathbf{f} & & X \\ \text { c, f, t } & X & X \\ \text { c, f, t } & X & X \\ \mathbf{t} & X & X \\ \text { c, f, t } & X & X \\ \mathbf{f} & & X \\ \mathbf{t} & & X \\ \text { c, f, t } & X & X \\ \text { c, f, t } & X & X \\ \text { c, f, t } & X & X \\ \mathbf{t} & & X\end{array}$

$\begin{array}{ccc}\mathbf{t} & & X \\ \mathbf{c}, \mathbf{f}, \mathbf{t} & X & X \\ \mathbf{f , t} & X & X\end{array}$

$\begin{array}{ccc}\mathbf{f}, \mathbf{t} & X & \\ \mathbf{t} & & X \\ \mathbf{c}, \mathbf{f}, \mathbf{t} & X & X \\ \mathbf{t} & & X \\ \mathbf{c , t} & X & X \\ \mathbf{f} & & X\end{array}$

\begin{tabular}{ccc}
$\mathbf{c , t}$ & $X$ & $X$ \\
$\mathbf{t}$ & & $X$ \\
$\mathbf{c , t}$ & & $X$ \\
$\mathbf{f}$ & $X$ & \\
$\mathbf{f , t}$ & $X$ & $X$ \\
$\mathbf{c , f} \mathbf{t}$ & $X$ & $X$ \\
$\mathbf{f , t}$ & $X$ & $X$ \\
$\mathbf{t}$ & & $X$ \\
$\mathbf{f , t}$ & $X$ & $X$ \\
$\mathbf{f , t}$ & $X$ & $X$ \\
$\mathbf{c , t} \mathbf{t} \mathbf{t}$ & $X$ & $X$ \\
$\mathbf{f , t}$ & $X$ & $X$ \\
$\mathbf{f , t}$ & $X$ & $X$ \\
$\mathbf{f}$ & $X$ & \\
$\mathbf{c}, \mathbf{f}, \mathbf{t}$ & $X$ & $X$ \\
$\mathbf{f}$ & $X$ & \\
\hline
\end{tabular}




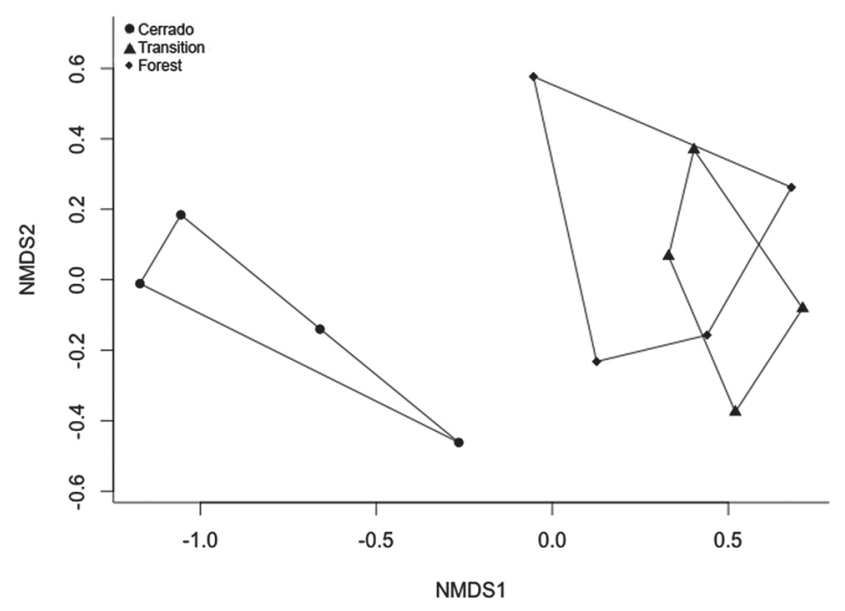

Figure 5. Non-metric multidimensional scaling (NMDS) of the butterfly species composition in sampling sites of Cerrado domain, Atlantic Rainforest and Forest-Cerrado transition areas, in the 'Serra do Rola-Moça' State Park, Minas Gerais state, Brazil.

erage of both seasons reached $80 \%$ (Fig. 4), but demonstrates that with more one year of sampling the coverage of species richness would tend to the estimated number. This suggests that the actual overlap between the faunas of the two seasons may be greater than the observed.

The species richness and composition of the butterfly assemblage did not vary significantly between the dry and the rainy seasons (GLM = Gaussian distribution, $p=0.79$ and ANOSIM, $p=0.49$ ). This result could be different maximizing the sample effort, either using bait trap for fruit-feeding species, and/or increasing the years of sampling. Pereira et al. (2017), for instance, collected for two years and found statistical differences in fruit-feeding butterfly composition between dry and rainy seasons. On the other hand, significant differences were found when the composition of butterfly assemblages was compared among sampling sites, (ANOSIM, $p=0.005$; Fig. 5), but not among their species richness (GLM = Gaussian distribution, $\mathrm{p}=0.065$ ).

Among the 311 recorded species, eight are probably new, of which seven belong each to one of the following genera: Chalcone, Cogia, Cumbre, Cymaenes, Lucida, Moneuptychia, Phlebodes. A probably new species of Hesperiinae, however, could not be identified even to genus level. This specimen was collected in the Ibirité municipality, in riparian forest, at the rainy season.

\section{Environmental variation}

The NMDS indicates that grassland and forest environments in the PESRM have distinct faunas. Most species restricted to the Cerrado domain (Brown Jr. \& Mielke, 1967b; Mielke et al., 2008, 2012) in our sample belong to Pyrginae (Hesperiidae) and Theclinae (Lycaenidae), especially to the genera Cogia and Strymon, respectively. According to the list presented by Mielke et al. (2012), the species of Pyrginae also sampled in this study Chiomara basigutta (Plötz, 1884), Cogia calchas (Herrich-Schäffer, 1869), Heliopetes omrina (Butler, 1870) and Viola viollela
(Mabille, 1898) have preference for natural grassland habitats. Moreover, Hesperiinae (Hesperiidae) and Satyrinae (Nymphalidae) are more abundant in grassland habitats (Casagrande et al., 2012), probably because larvae of Hesperiinae feed exclusively on monocots (Carneiro et al., 2014), and those of Satyrinae almost exclusively on monocots (Peña \& Wahlberg, 2008), which are poorly represented in the forest environment. Despite the fact that those feeding habits justify their abundance in the Cerrado vegetation, in the PESRM, almost $50 \%$ of the species of Hesperiinae and $30 \%$ of the Satyrinae were found only in the transition areas.

More attention has been given to the butteflies of the Atlantic Rainforest than to those of the Cerrado (Brown Jr. \& Freitas, 2000; Uehara-Prado et al., 2007; Carneiro et al., 2008). However, as emphasized by Dolibaina et al. (2011) and Carneiro et al. (2014), the characteristic species of natural grasslands must also be considered conservation priorities since this vegetation type is either under threat.

\section{New record, endemic and threatened species}

Among the species sampled in the PESRM, Hypoleria lavinia (Hewitson, [1855]) was known, until now, only from the Parque Estadual do Rio Doce (Silva et al., 2010) and from Viçosa (Cruz et al., 2012), both in the core of the Atlantic Rainforest, in the state of Minas Gerais. This is, therefore, a new record for the Quadrilátero Ferrífero. Panoquina peraea (Hewitson, 1866), previously known to occurs only in the states of Rio de Janeiro (Mielke \& Casagrande, 2002) and Bahia (Lima \& Zacca, 2014), is a new record for Minas Gerais.

Actinote quadra (Schaus, 1902) (Nymphalidae), classified as vulnerable in the Red Book of Brazilian Threatened Fauna (Machado et al., 2008), was sampled in the rock field at an altitude of about 1,475 m (2003'29"S, $44^{\circ} 00^{\prime} 06^{\prime \prime} \mathrm{W}$; municipality of Brumadinho, Minas Gerais). This new record for the species and other three records are best detailed in Gomes et al. (2014). This species occurs in isolated populations in mountain regions in the states of Minas Gerais, São Paulo and Rio de Janeiro, especially in the Serra da Mantiqueira, usually in wet forests above 1,000 m (Freitas et al., 2009; Freitas \& Marini-Filho, 2011). Main threats to Actinote quadra are habitat degradation and destruction (Freitas \& Marini-Filho, 2011) and this reinforces the importance of the PESRM for the preservation of the species, as well as for all species of this genus inhabiting the park, since they are all endemic of mountainous environments and frequently rare (Gomes et al., 2014).

Eight species endemic or potentially endemic of the Cerrado domain, according to Mielke et al. (2008) and Pinheiro et al. (2010), were also found in the PESRM: Yphthimoides straminea (Butler, 1867), Cogia grandis Riley, 1921, Cogia cerradicola (Mielke, 1967), Lerema veadeira Mielke, 1968, Parides bunichus diodorus (Hopffer, 1865), Pseudoscada acilla quadrifasciata Talbot, 1928, Sarbia catomelaena Mabille \& Boullet, 1908 and Sophista latifas- 
ciata (Spitz, 1930). However, some of them were found in the forest and/or transition environments. In fact, Murray \& Prowell (2005) pointed out that many species of the genus Yphthimoides are endemic of the Brazilian Southeast Region and Freitas et al. (2012) indicated that most of them are found both in the Atlantic Rainforest and in the Cerrado domain. This is due to the isolated forests patches in the Cerrado, as well as riparian forests that are found along the rivers in the Cerrado domain (Silva \& Bates, 2002; Werneck, 2011). This means that butterflies species in the Cerrado domain can also inhabit forests.

The geological formation of the PESRM is very peculiar, composed by ferruginous field, savanna and forest. This is one of the few parks in Brazil where this rare environment is preserved. This and the fact that those fields are the natural habitat for many species of butterflies make the PESRM an especially important conservation area. The knowledge about the rich fauna of butterflies of Minas Gerais, mainly the new records and possible new species as well as endangered species provides important information for preservation actions and environmental conservation, especially on places with such a peculiar environment.

\section{ACKNOWLEDGEMENTS}

We are grateful to Diego Rodrigo Dolibaina (UFPR), André Victor Lucci Freitas and Lucas Augusto Kaminski (UNICAMP) for the identification of part of the material, and Marco Paulo Macedo Magalhães for checking the identification of some specimens. We thank Alessandro Lima, Cássio Montes, Felipe Freitas, Glória Soares, Isabela Oliveira, José Eustáquio, Mércia Araújo, Naíla Fernandes, Rayane Melo, Rodolfo Arantes, Stanley Franco, Thaís Cardoso and Walter Ávila for field assistance. We also thank Marina do Vale Beirão and Frederico Kirst for the help with statistical analyses, and Luciana Barçante Ferreira for critically reviewing this manuscript.

\section{REFERENCES}

Brown Jr., K.S. 1992. Borboletas da Serra do Japi: diversidade, habitats, recursos alimentares e variação temporal. In: Patrícia, L. \& Morellato, C. (Eds.). História Natural da Serra do Japi: Ecologia e preservação de uma área florestal no Sudeste do Brasil. Campinas, FAPESP. p. 142-186.

Brown Jr., K.S. \& Freitas, A.V.L. 2000. Atlantic rainforest Butterflies: indicators for landscape conservation. Biotropica, 32: 934-956.

Brown Jr., K.S. \& Mielke, 0.H.H. 1967a. Lepidoptera of the Central Brazil Plateau. I. Preliminary list of Rhopalocera: introduction, Nymphalidae, Libytheidae. Journal of the Lepidopterists' Society, 21(2): 77-106.

Brown Jr., K.S. \& Mielke, 0.H.H. 1967b. Lepidoptera of the Central Brazil Plateau. I. Preliminary list of Rhopalocera (continued): Lycaenidae, Pieridae, Papilionidae, Hesperiidae. Journal of the Lepidopterists' Society, 21(3): 145-168.

Brown Jr., K.S. \& Mielke, 0.H.H. 1968. Lepidoptera of the Central Brazil Plateau. III. Partial list for the Belo Horizonte area, showing the character of the south-eastern "blend zone". Journal of the Lepidopterists' Society, 22(3): 147-157.
Carneiro, E.; Mielke, 0.H.H. \& Casagrande, M.M. 2008. Inventários de borboletas no Brasil: estado da arte e modelo de áreas prioritárias para pesquisa com vista à conservação. Natureza e Conservação, 6(2): 68-90.

Carneiro, E.; Mielke, 0.H.H.; Casagrande, M.M. \& Fiedler, K. 2014. Community Structure of Skipper Butterflies (Lepidoptera, Hesperiidae) along Elevational Gradients in Brazilian Atlantic Rainforest Reflects Vegetation Type Rather than Altitude. Plos One, 9: 1-11.

Casagrande, M.M.; Dolibaina, D.R.; Carneiro, E.; Dias, F.M.S.; Leite, L.A.R. \& Mielke, 0.H.H. 2012. Borboletas (Hesperioidea e Papilionoidea) de Jaguariaíva, Paraná, Brasil: Inventário em um enclave de Cerrado meridional. In: Carpanezzi, 0.T.B. \& Campos, J.B. (Eds.). Coletânea de Pesquisas. Parques Estaduais de Vila Velha, Cerrado e Guartelá. Curitiba, Instituto Ambiental do Paraná. p. 295-308.

Crawley, M.J. 2013. The R Book. London, John Wiley and Sons Ltd.

Cruz, K.C.; Lelis, S.M.; Godinho, M.A.S.; Fonseca, R.S.; Ferreira, P.S.F. \& Vieira, M.F. 2012. Species richness of anthophilous butterflies of an Atlantic Rainforest fragment in Southeastern. Revista Ceres, Viçosa, 59: 571-579.

D'Abrera, B. 2005. World Butterflies. Hill House Publishers, London. (Reduced version of The Concise Atlas of the Butterflies of the World, 2001).

Dolibaina, D.R.; Mielke, 0.H.H. \& Casagrande, M.M. 2011. Borboletas (Papilionoidea e Hesperioidea) de Guarapuava e arredores, Paraná, Brasil: um inventário com base em 63 anos de registros. Biota Neotropica, 11: 1-14.

Drummond, G.M. \& Martins, C.S. (Coord.). 2007. Plano de manejo do Parque Estadual da Serra do Rola Moça. Belo Horizonte. Available at: http://www. biodiversitas.org.br/planosdemanejo/pesrm/regiao.htm. Access in: 02/07/2016.

Ebert, H. 1969. On the frequency of butterflies in eastern Brazil, with a list of the butterfly fauna of Poços de Caldas, Minas Gerais. Journal of the Lepidopterists'Society, 23(3): 1-48.

Emery, E.0.; Brown Jr., K.S. \& Pinheiro, C.E.G. 2006. As borboletas (Lepidoptera, Papilionoidea) do Distrito Federal, Brasil. Revista Brasileira de Entomologia, 50: 85-92.

Freitas, A.V.L. \& Marini-Filho, 0.J. 2011. Plano de ação nacional para conservação dos Lepidópteros ameaçados de extinção. Brasília, ICMBio. (Série Espécies Ameaçadas).

Freitas, A.V.L.; Francini, R.B. \& Souza, T.S. 2009. Immature stages and natural history of the threatened butterfly Actinote quadra (Nymphalidae: Heliconiinae: Acraeini). Tropical Lepidoptera Research, 19: 83-88.

Freitas, A.V.L; Kaminski, L.A.; Mielke, O.H.H.; Barbosa, E.B. \& Silva-Brandão, K.L. 2012. A new species of Yphthimoides (Lepidoptera: Nymphalidae: Satyrinae) from the southern Atlantic Rainforest region. Zootaxa, 3526: 31-44.

Garwood, K.; Lehman, R.; Carter, W. \& Carter, G. 2009. Butterflies of Southern Amazon: A photography checklist of common species. Texas, McAllen, RiCalé Publishing.

Gomes, V.; Lourenço, G.M.; Soldati, D.; Iserhard, C.A.; Souza, T.S.; Kaminski, L.A. \& Freitas, A.V.L., 2014. New geographical records for the threatened butterfly Actinote quadra (Lepidootera: Nymphalidae: Heliconiinae). Journal of the Lepidopterists' Society, 68: 289-292.

Iserhard, C.A. 2011. Padronização dos métodos de captura de lepidópteros com puçá. In: Encontro Pan Lepidópteros e RedeLep. Brasília.

Iserhard, C.A.; Brown Jr., K.S. \& Freitas, A.V.L. 2013. Maximized sampling of butterflies to detect temporal changes in tropical communities. Journal of Insect Conservation, 17: 615-622.

Iserhard, C.A.; Uehara-Prado, M.; Marini-Filho, 0.J.; Duarte, M. \& Freitas, A.V.L. 2018. Fauna da Mata Atlântica: Lepidoptera-borboletas. In: MonteiroFilho, E.L.A \& Conte, C.E. (Orgs.). Revisões em zoologia da Mata Atlântica. Curitiba, Editora UFPR. p. 57-102.

Lamas, G. 2004. Atlas of Neotropical Lepidoptera. Checklist: Part 4A. Hesperioidea - Papilionoidea. Gainesville, Scientific Publishers. 
Lewinsohn, T.M.; Freitas, A.V.L. \& Prado, P.I. 2005. Conservação de invertebrados terrestres e seus habitats no Brasil. Megadiversidade, 1: 62-69.

Lima, J.N.R. \& Zacca, T. 2014. Lista de Espécies de Borboletas (Lepidoptera: Hesperioidea e Papilionoidea) de uma Área de Semiárido na Região Nordeste do Brasil. EntomoBrasilis, 7: 33-40.

Machado, A.B.M., Drummond, G.M. \& Paglia, A.P. 2008. Livro vermelho da fauna brasileira ameaçada de extinção. Volume 1. MMA, Brasília. Belo Horizonte, Fundação Biodiversitas.

Mielke, O.H.H. \& Casagrande, M.M. 1997. Papilionoidea e Hesperioidea (Lepidoptera) do Parque Estadual do Morro do Diabo, Teodoro Sampaio, São Paulo, Brasil. Revista Brasileira de Zoologia, 14: 967-1001.

Mielke, 0.H.H. \& Casagrande, M.M. 2002. Notas taxonômicas em Hesperiidae neotropicais, com descrições de novos taxa (Lepidoptera). Revista Brasileira de Zoologia, 19(1): 27-76.

Mielke, 0.H.H.; Carneiro, E. \& Casagrande, M.M. 2012. Hesperiidae (Lepidoptera, Hesperioidea) from Ponta Grossa, Paraná, Brazil: 70 years of records with special reference to faunal composition of Vila Velha State Park. Revista Brasileira de Entomologia, 56(1): 59-66.

Mielke, 0.H.H.; Emery, E.0. \& Pinheiro, C.E.G. 2008. As borboletas Hesperiidae (Lepidoptera, Hesperioidea) do Distrito Federal, Brasil. Revista Brasileira de Entomologia, 52: 283-288.

Mittermeier, R.A.; Gil, P.R.; Hoffmann, M.; Pilgrim, J.; Brooks, T.; Mittermeier, C.G.; Lamoreux, J. \& Da Fonseca, G.A.B. 2004. Hotspots revisited: Earth's biologically richest and most endangered terrestrial ecoregions. Agrupación Sierra Madre, Conservation International.

Motta, P.C. 2002. Butterflies from the Uberlândia region, Central Brazil: Species list and biological comments. Brazilian Journal of Biology, 62: 151-163.

Murray, D.L. \& Prowell, D.P. 2005. Molecular phylogenetics and evolutionary history of the neotropical satyrine subtribe Euptychiina (Nymphalidae: Satyrinae). Molecular Phylogenetics and Evolution, 34: 67-80.

Peña, N. \& Wahlberg, C. 2008. Prehistorical climate change increased diversification of a group of butterflies. Biology Letters, 4: 274-278.

Penz, C.M. \& Devries, P.J. 2002. Phylogenetic analysis of Morpho Butterflies (Nymphalidae, Morphinae): Implications for classification and natural history. American Museum Novitates, 3374: 1-33.

Pereira, G.C.N.; Coelho, M.S.; Beirão, M.V.; Braga, R.F. \& Fernandes, G.W. 2017. Diversity of fruit-feeding butterflies in a mountaintop archipelago of rainforest. Plos One, 12(6): 1-20.

Pinheiro, C.E.G.; Malinov, I.K.; Emery, E.0. \& Schmidt, K. 2010. Endemismos e conservação de borboletas (Lepidoptera: Papilionoidea e Hesperioidea) no bioma Cerrado. In: Diniz, I.V., Filho, J.M., Machado, R.B. \& Cavalcanti, R.B. (Org.). Cerrado: conhecimento científico quantitativo como subsidio para as ações de conservação. Brasília, Thesaurus. p. 223-238.

$R$ Core Team. 2014. R: A language and environment for statistical computing. Vienna, R Foundation for Statistical Computing. Available at: http:// www.R-project.org. Access in: 14/03/2016.
RStudio Team. 2015. RStudio: Integrated development for $R$ (Version 1.1.453). Boston, MA. Available at: http://www.rstudio.com. Access in: 06/07/2018.

Santos, J.P.; Iserhard, C.A.; Teixeira, M.0. \& Romanowski, H.P. 2011. Fruitfeeding butterflies guide of subtropical Atlantic Rainforest and Araucaria Moist Forest in State of Rio Grande do Sul, Brazil. Biota Neotropica, 11: 253-274.

Seraphim, N.; Kaminski, L.A.; Devries, P.J.; Penz, C.; Callaghan, C.; Wahlberg, N.; Silva-Brandão, K.L. \& Freitas, A.V.L. 2018. Molecular phylogeny and higher systematics of the metalmark butterflies (Lepidoptera: Riodinidae). Systematic Entomology, 43: 407-425.

Silva, A.R.M.; Guimarães, M.P.M.; Vitalino, R.F.; Bagni, A.S.; Martins, Y.E.; Cordeiro, A.M. \& Oliveira, E.G. 2010. Borboletas frugívoras do Parque Estadual do Rio Doce/MG. MG.BIOTA, 3(4): 5-21.

Silva, J.M.C. \& Bates, J.M. 2002. Biogeographic patterns and conservation in the South American Cerrado: a tropical savanna hotspot. BioScience, 52(3): 225-234

Tabarelli, M.; Pinto, L.P.; Silva, J.M.; Hirota, M.M. \& Bedê, L.C. 2005. Desafios e oportunidades para a conservação da biodiversidade na Mata Atlântica brasileira. Megadiversidade, 1: 132-138.

Uehara-Prado, M.; Brown Jr., K.S \& Freitas, A.V.L. 2007. Species richness, composition and abundance of fruit-feeding butterflies in the Brazilian Atlantic Forest: comparison between a fragmented and a continuous landscape. Global Ecology and Biogeography, 16: 43-54.

Uehara-Prado, M.; Freitas, A.V.L.; Francini, R.B. \& Brown Jr., K.S. 2004. Guia das Borboletas Frugívoras da Reserva Estadual do Morro Grande e da Região de Caucaia do Alto, Cotia (São Paulo). Biota Neotropica, 4: 1-25.

Wahlberg, N.; Leneveu, J.; Kodandaramaiah, U.; Peña, C.; Nylin, S.; Freitas, A.V.L. \& Brower, A.V.Z. 2009. Nymphalid butterflies diversify following near demise at the Cretaceous/Tertiary boundary. Proceedings of the Royal Society B: Biological Sciences, 276: 4295-4302.

Warren, A.D.; Davis, K.J.; Stangeland, E.M.; Pelham, J.P. \& Grishin, N.V. 2017. Illustrated Lists of American Butterflies. Available at: http://www. butterfliesofamerica.com. Access in: 18/06/2018.

Warren, A.D.; Ogawa, J.R. \& Brower, A.V.Z 2009. Revised classification of the family Hesperiidae (Lepidoptera: Hesperioidea) based on combined molecular and morphological data. Systematic Entomology, 34: 467-523.

Warren, A.D.; Ogawa, J.R. \& Brower, A.V.Z. 2008. Phylogenetic relationships of subfamilies and circumscription of tribes in the family Hesperiidae (Lepidoptera: Hesperioidea). Cladistics, 24: 642-676.

Werneck, F.P. 2011. The diversification of eastern South American open vegetation biomes: Historical biogeography and perspectives. Quaternary Science Reviews, 30: 1630-1648.

Willmott, K.R. 2003. The genus Adelpha: Its systematics, biology and biogeography (Lepidoptera: Nymphalidae: Limenitidini). Gainesville, Scientific Publishers. 